\title{
Síndrome do ápice orbitário causada por herpes zóster oftálmico: relato de caso e revisão da literatura
}

\section{Herpes zoster ophthalmicus and orbital apex syndrome: case report and literature review}

Kenzo Hokazono', Marcelo Oliveira', Frederico Castelo Moura², Mário Luiz Ribeiro Monteiro³

\section{RESUMO}

O Herpes Zoster Oftálmico (HZO) decorre da infecção pelo vírus da varicela-zoster que permanece latente no gânglio de Gasser até que seja reativado e comprometa a divisão oftálmica do nervo trigêmeo. HZO freqüentemente causa manifestações oftalmológicas como lesões vesiculares palpebrais, ceratoconjuntivite, esclerite, uveíte, paralisia oculomotora, miosite orbitária e neurite óptica. Raramente o acometimento do ápice da órbita pode ser a manifestação inicial desta grave afecção. Este trabalho relata um caso de síndrome do ápice orbitário associado à meningite, causado por HZO e que foi tratado com corticosteróide e aciclovir sistêmicos.

Descritores: Herpes zoster oftálmico/complicações; Síndrome do ápice orbitário; celulite orbitária; Oftalmoplegia; Neurite óptica; Relatos de casos

\footnotetext{
${ }^{1}$ Residentes do Departamento de Oftalmologia e Otorrinolaringologia da Faculdade de Medicina da Universidade de São Paulo USP - São Paulo (SP) - Brasil.

2Doutor, Médico do Setor de Neuroftalmologia e Doenças da Órbita do Departamento de Oftalmologia e Otorrinolaringologia da Faculdade de Medicina da Universidade de São Paulo -USP - São Paulo (SP) - Brasil.

${ }^{3}$ Livre-docente, Professor Associado do Departamento de Oftalmologia e Otorrinolaringologia da Faculdade de Medicina da Universidade de São Paulo - USP- São Paulo (SP) - Brasil.

Trabalho desenvolvido na Divisão de Oftalmologia do Hospital das Clínicas da Faculdade de Medicina da Universidade de São Paulo (USP), São Paulo (SP) - Brasil.
} 


\section{INTRODUÇÃO}

$\mathbf{O}$ Herpes zoster ocorre devido à reativação tardia de uma infecção pelo vírus da varicelazoster. Embora se manifeste principalmente nos dermátomos sensoriais torácicos, pode também acometer os nervos cranianos, como a divisão oftálmica do nervo trigêmeo, quando é denominado herpes zoster oftálmico (HZO), e que ocorre em aproximadamente 10\% dos casos. Embora várias complicações oftalmológicas decorrentes da infecção pelo vírus varicela-zoster já tenham sido descritas, oftalmoplegia completa e perda visual são manifestações iniciais raras. ${ }^{(1)}$

Síndrome do ápice orbitário consiste de sinais de envolvimento das estruturas nervosas que atravessam o forame óptico e a fissura orbitária superior comprometendo os nervos oculomotor, troclear, abducente e da divisão oftálmica do nervo trigêmeo, juntamente com acometimento do nervo óptico. ${ }^{(2)}$ Pode ter várias etiologias incluindo: afecção neoplásica, vascular, traumática, infecciosa ou inflamatória. ${ }^{(3-8)}$

O objetivo deste é relatar a associação rara entre a síndrome do ápice orbitário e a meningite secundária à infecção pelo vírus varicela-zoster, além de revisar outros casos na literatura, enfatizando a importância da identificação precoce do acometimento do nervo óptico e discutindo os mecanismos fisiopatogênicos envolvidos, bem como o tratamento desta afecção.

\section{Relato do caso}

Paciente do sexo masculino, 19 anos, branco, previamente hígido, apresentou quadro de dor lancinante
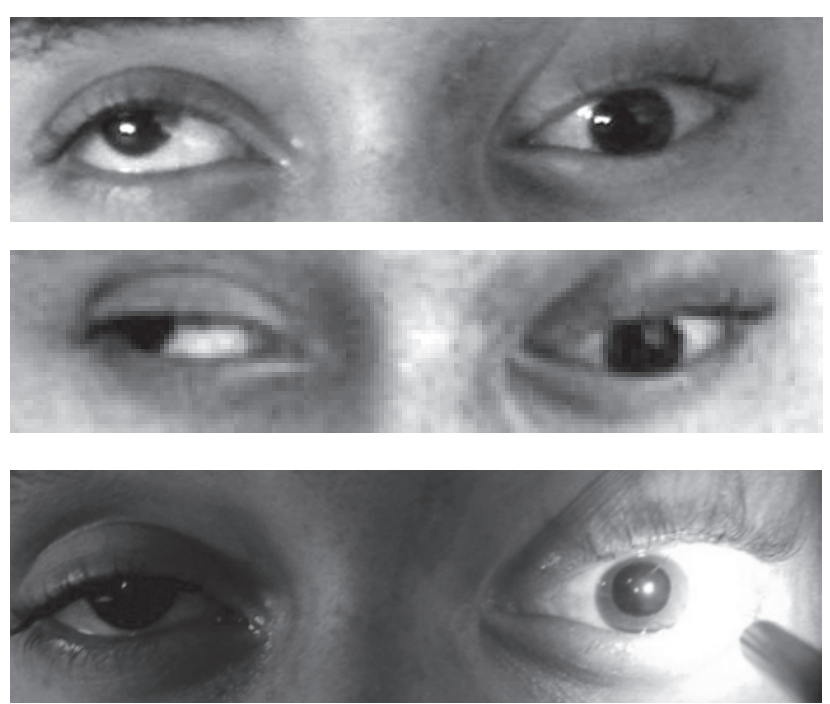

na hemiface esquerda há 15 dias. Cinco dias após o início da dor, houve aparecimento de eritema vesicular e edema periorbital na região inervada pelo ramo oftálmico do nervo trigêmeo. Evoluiu com perda visual e ptose à esquerda, associadas à sonolência, cefaléia, anorexia e emagrecimento. Negava febre e outros sintomas.

Ao exame externo, o paciente apresentava eritema máculo-papular, com crostas hemáticas e raras vesículas na hemiface esquerda, envolvendo a ponta do nariz. Ao exame ocular, apresentava acuidade visual de 1,0 no olho direito (OD) e de conta-dedos a 10 centímetros no olho esquerdo (OE). A pupila do OE era discretamente maior que a do OD e havia diminuição da resposta fotomotora direta no $\mathrm{OE}$ e defeito aferente relativo importante à esquerda. Ao exame da motilidade ocular extrínseca foi observada oftalmoplegia completa a esquerda, inclusive ptose palpebral (Figura 1). A exoftalmometria foi de 19 e $23 \mathrm{~mm}$, no OD e OE, respectivamente. A sensibilidade corneana e cutânea da fronte estavam diminuídas à esquerda. Ao exame de biomicroscopia do segmento anterior, havia hiperemia e quemose conjuntival discreta, edema de córnea discreto e células inflamatórias (duas cruzes) na câmara anterior. A tonometria de aplanação (Goldmann) foi de $14 \mathrm{e} 8 \mathrm{mmHg}$ respectivamente à direita e esquerda. À oftalmoscopia revelou fundo-de-olho normal em ambos os olhos, sem alterações do nervo óptico ou sinais de vasculite. $\mathrm{O}$ exame neurológico era normal, exceto pelas alterações ocasionadas pelo HZO.

O hemograma completo, velocidade de hemossedimentação e proteína $\mathrm{C}$ reativa eram normais. As sorologias para HIV e hepatite $\mathrm{C}$ foram negativas.
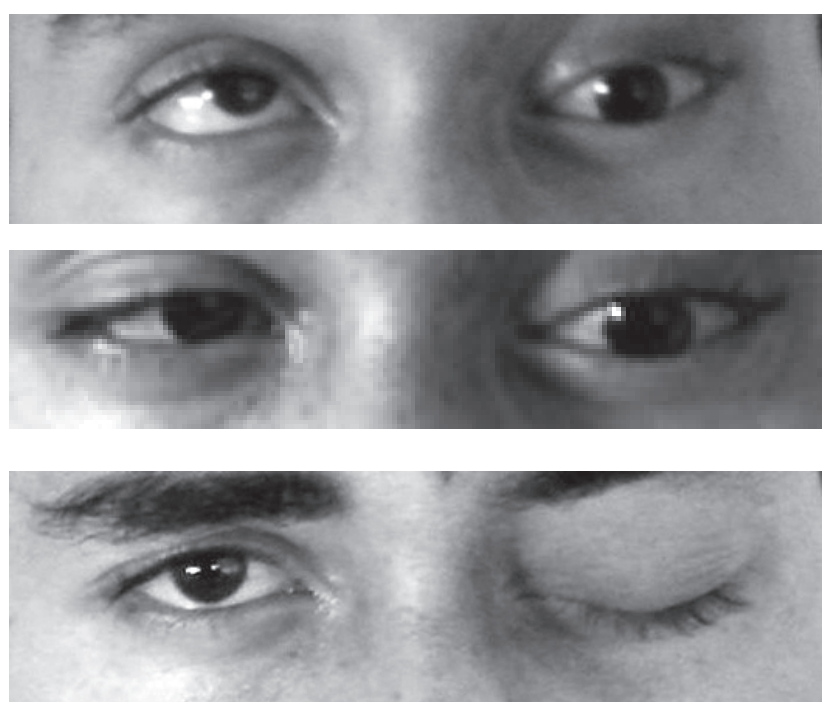

Figura 1. Exame externo e da motilidade ocular extrínseca evidenciando oftalmoplegia completa, com midríase, paralisia da musculatura ocular extrínseca e ptose palpebral. 

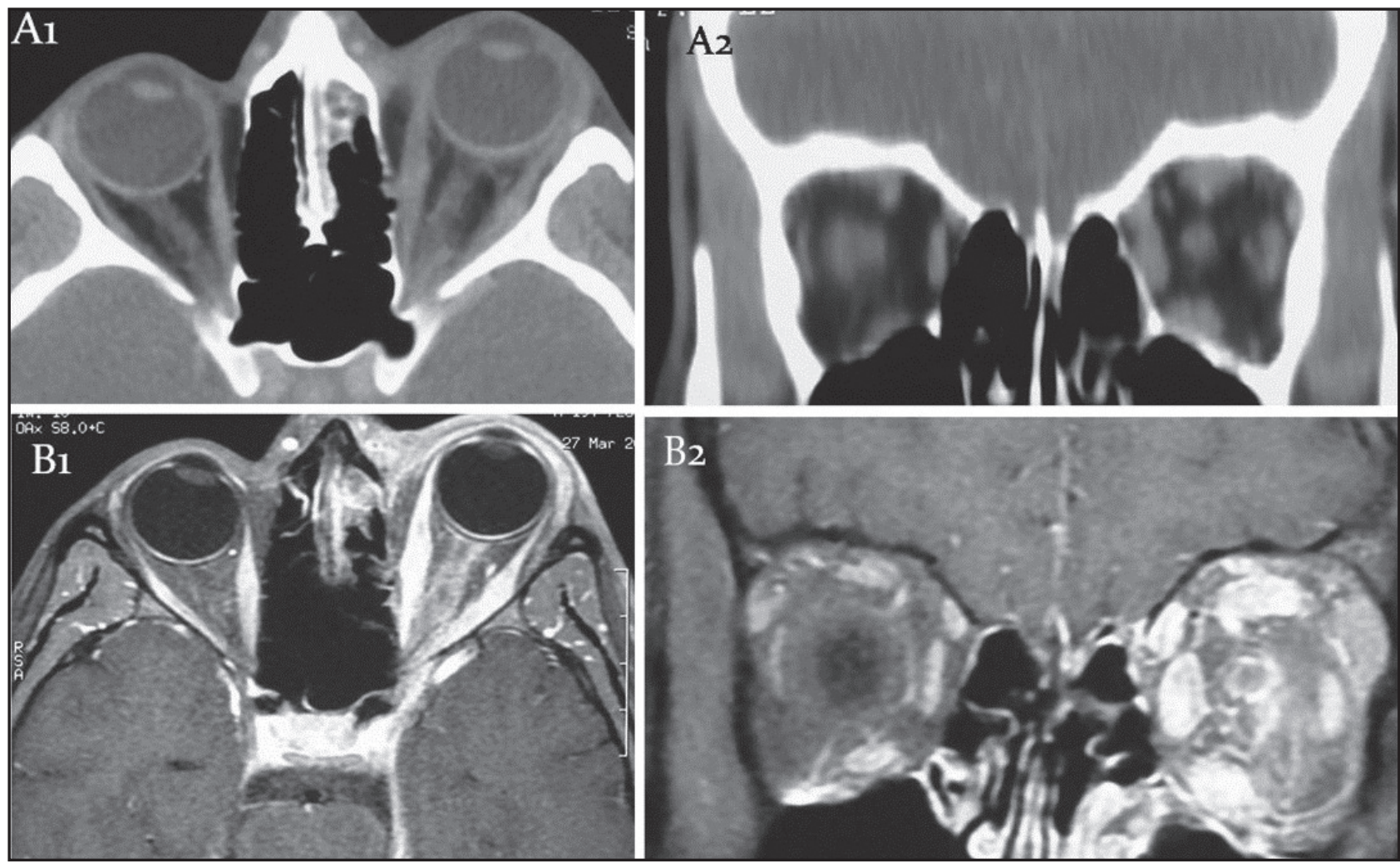

Figura 2. Exames de tomografia computadorizada (A1 e A2) e de imagem por ressonância magnética (B1 e B2) evidenciando o espessamento dos músculos extra-oculares, realce do nervo óptico e espessamento e realce meníngeo.

Foi realizada tomografia computadorizada (TC) de órbitas e seios da face com cortes axiais e coronais demonstrando espessamento difuso da musculatura ocular extrínseca. A ressonância magnética (RM) de crânio e órbitas evidenciou espessamento dos músculos extrínsecos da órbita esquerda, com aumento do sinal de impregnação da musculatura ocular extrínseca esquerda, em imagens enfatizando T2; tênue halo de realce no nervo óptico esquerdo após administração de contraste, realce leptomeníngeo na base do crânio (sugerindo inflamação meníngea regional) e assimetria de seios cavernosos, maior à esquerda (indicativo de congestão) (Figura 2). A análise do líquor ,extraído por punção lombar, mostrou pressão de abertura normal, proteínas $=149 \mathrm{mg} / \mathrm{dl}$, glicose $=32 \mathrm{mg} / \mathrm{dl}($ glicemia $=63 \mathrm{mg} /$ dl), células $=418 / \mathrm{mm}^{3}$ (linfócitos $=88 \%$ ).

$\mathrm{O}$ diagnóstico de $\mathrm{HZO}$ foi feito baseado no quadro clínico, laboratorial e radiológico. O tratamento, instituído em regime de internação hospitalar, consistiu de aciclovir $10 \mathrm{mg} / \mathrm{kg} /$ dose a cada 8 horas e pulsoterapia com metilprednisolona intravenoso $500 \mathrm{mg} /$ dia por 3 dias, seguido de prednisona $1 \mathrm{mg} / \mathrm{kg} /$ dia via oral com redução gradual de acordo com a remissão dos sinais e sintomas, além de tratamento tópico, utilizando colírios de dexametasona $0,1 \%$ e tropicamida $1 \%$ no olho esquerdo. No $3^{\circ}$ dia de internação houve melhora da acuidade visual de conta-dedos para 0,3 , associada à redução do defeito pupilar aferente relativo. A melhora da ptose e da motilidade ocular extrínseca foi mais lenta, com abertura palpebral parcial e discreta recuperação da abdução após 4 semanas. No $4^{\circ}$ mês de seguimento, o paciente apresentava acuidade visual de 1,0 em ambos os olhos, ausência de ptose palpebral e rotações binoculares normais, exceto por adução reduzida do olho esquerdo (Figura 3 ).

\section{Discussão}

O vírus varicela-zoster (VVZ) é um membro da família dos herpes vírus que provoca duas doenças clinicamente distintas: a varicela e o Herpes zoster. O Herpes zoster é decorrente da reativação do VVZ que se encontrava latente, e se manifesta como erupção vesiculosa aguda e dolorosa em dermátomos, precedidas por pródromos de febre e cefaléia. O Herpes zoster oftálmico (HZO) é decorrente da reativação do vírus varicela-zoster que se encontrava latente no gânglio trigeminal, ${ }^{(9)}$ resultando no comprometimento dos ra- 


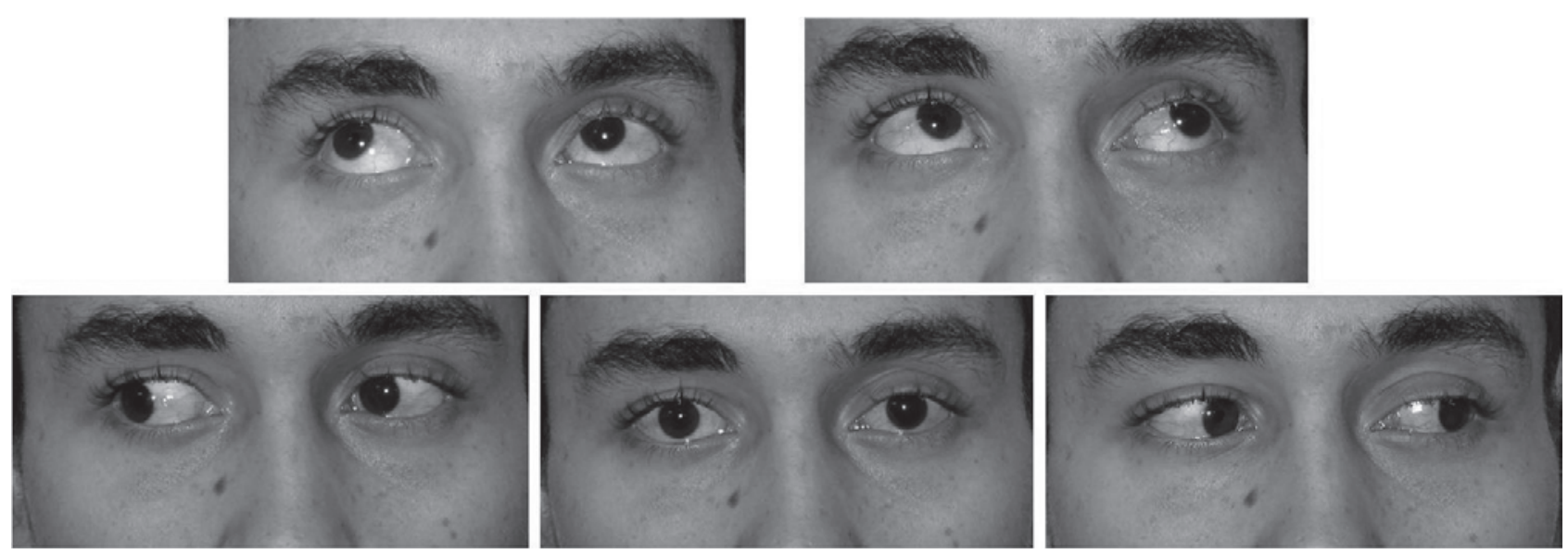

Figura 3: Exame da motilidade ocular extrínseca do paciente com herpes zoster oftálmico, mostrando recuperação total da oftalmoplegia 4 meses após a apresentação inicial

mos da divisão oftálmica do nervo trigêmeo, dos quais o ramo frontal é acometido mais frequentemente. ${ }^{(1)} \mathrm{HZO}$ é responsável por 10-20\% dos casos de Herpes zoster e o envolvimento ocular ocorre em aproximadamente $50 \%$ dos casos. ${ }^{(9)}$ Várias manifestações oftalmológicas associadas ao HZO já foram relatadas, como: erupções vesiculares palpebrais, ceratoconjuntivite, esclerite e uveíte. O HZO também pode causar complicações neuro-oftalmológicas, sendo as mais comuns são as síndromes oftalmoplégicas isoladas. Marsh e cols. ${ }^{(10)}$ relatam paralisia de músculo ocular externo em $29 \%$ dos pacientes examinados. ${ }^{(10)}$

Dentre os nervos da motricidade ocular, o oculomotor (III nervo craniano) é o mais comumente acometido, seguido pelo nervo abducente (IV nervo craniano). Além disso, neurite óptica é uma rara complicação do HZO que pode causar perda visual associada à oftalmoplegia. ${ }^{(11)}$ Há dois casos de neurite óptica associada à $\mathrm{HZO}$, confirmados por RM, um deles se apresentando com edema de disco óptico e o outro como neurite óptica retrobulbar. ${ }^{(12)}$

No caso aqui descrito, o paciente não apresentou a forma mais usual de manifestação oftalmológica do HZO a qual seria o acometimento isolado um nervo oculomotor, mas sim uma forma incomum e mais grave, com paralisia de todos os pares cranianos que inervam a musculatura extrínseca ocular, manifestado clinicamente como oftalmolplegia completa, ptose palpebral e anisocoria. $\mathrm{O}$ acometimento simultâneo de todos os nervos oculomotores associados à perda visual deve nos indicar o diagnóstico de síndrome do ápice orbitário.

Síndrome do ápice orbitário é definida pela associação de neuropatia óptica, paralisia completa ou parcial dos nervos da motricidade ocular e parestesia na distribuição dos ramos (oftálmico e, às vezes, maxilar) do nervo trigêmeo e a associação com HZO é rara, havendo descritos dois casos de síndrome do ápice orbitário secundário ao $\mathrm{HZO}$, associados à meningoencefalite. ${ }^{(8)}$. Similarmente, o paciente aqui descrito apresentava sonolência, cefaléia e sinais de acometimento meníngeo, como aumento do número de células no líquor e realce radiológico da meninge na base do crânio (Figura 2) Recentemente, foi descrito o caso de uma paciente de 63 anos, que apresentou HZO à direita, associado à perda visual e discromatopsia devido à neurite óptica, retrobulbar, oftalmoplegia completa, ptose, proptose e uveíte anterior. ${ }^{(2)}$

É importante acrescentar que o paciente aqui relato tinha uveíte anterior leve e não apresentava alterações retinianas ou do nervo óptico. Entretanto, apresentava perda importante da função visual e defeito pupilar aferente relativo, o que nos levou a suspeitar que havia também acometimento da via óptica anterior, já que as alterações oculares não justificariam a redução da acuidade visual e o defeito pupilar aferente relativo.

A fisiopatogenia do envolvimento do nervo óptico e dos nervos oculomotores é controversa e vários mecanismos já foram propostos, como o acometimento das estruturas nervosas da fissura orbitária superior (III, IV, $\mathrm{V}$ e VI pares cranianos), ${ }^{(9)}$ decorrente das perineurite $\mathrm{e}$ perivasculite intensas ocasionadas pelo VVZ, através de uma reação de hipersensibilidade imuno-mediada. Outra explicação para a ocorrência de oftalmoplegia no HZO é a intensa inflamação dos músculos extra-oculares, sem envolvimento dos nervos oculomotores, ${ }^{(13)} \mathrm{em}$ bora a oftalmoplegia raramente se manifeste de forma completa na miosite orbitária. Em nosso paciente, a primeira hipótese é mais bem aceita devido à presença de 
oftalmoplegia e ptose completa, o que torna improvável que o mecanismo tenha sido pelo acometimento dos músculos extraoculares.

O acometimento do nervo óptico pode se manifestar na forma de neurite óptica anterior com edema do disco óptico ou de neurite retrobulbar, ou mesmo, já se apresentar com atrofia do nervo óptico. Foi descrito um caso de neuropatia óptica secundária a herpes zoster oftálmico em uma paciente de $48 \operatorname{anos}^{(14)}$, que apresentou perda visual súbita no olho esquerdo, após um mês do episódio de Herpes zoster oftálmico, apenas com percepção luminosa já no início do quadro, sem resposta ao tratamento com corticoterapia. Após três meses, desenvolveu palidez temporal do nervo óptico esquerdo. A perda visual do nosso paciente provavelmente foi causada por neurite óptica retrobulbar, devido aos achados de defeito pupilar aferente relativo e fundoscopia normal, tendo em vista que as alterações corneanas e a uveíte anterior não justificavam a gravidade da perda visual. $\mathrm{O}$ realce meníngeo observado à imagem por RM também é uma evidência importante de acometimento inflamatório que provavelmente incluiu o nervo óptico. Finalmente, no presente caso, a melhora visual importante indica que o acometimento do nervo óptico foi inflamatório e não isquêmico. ${ }^{(15)}$

Em conclusão, este relato foi feito para ressaltar a rara associação entre $\mathrm{HZO}$ e síndrome do ápice orbitário e enfatizar a importância de se determinar a presença de perda visual nesta afecção, usando o exame neurooftalmológico e de imagem, assim como a evolução favorável quando o tratamento da neurite óptica é instituído precocemente, com melhora do prognóstico visual.

\section{Abstract}

Herpes Zoster ophthalmicus (HZO) is caused by a varicella-zoster virus infection which remains latent in the ganglion of Gasser until it is reactivated and compromise the ophthalmic division of the trigeminal nerve. HZO commonly causes neuro-ophthalmic complications such as vesicular lesions in the eyelids, keratoconjunctivitis, sclertis, uveitis, ocular palsy, orbital miositis and optic neuritis. HZO rarely presents as an orbital apex syndrome. This paper describes a patient with of orbital apex syndrome associate and meningitis caused by HZO which was treated with systemic steroids and acyclovir.

Keywords: Herpes zoster ophthalmicus/ complications; Orbital apex syndrome; Orbital cellulitis; ophthalmoplegia; Optic neuritis; Case reports

\section{REFERÊNCIAS}

1. Liesegang TJ. Herpes zoster ophthalmicus natural history, risk factors, clinical presentation, and morbidity. Ophthalmology. 2008;115(2 Suppl):S3-12.

2. Bourke RD, Pyle J. Herpes zoster ophthalmicus and the orbital apex syndrome. Aust N Z J Ophthalmol. 1994;22(1):77-80.

3. Coppeto JR, Yamase H, Monteiro ML. Chronic ophthalmic Wegener's granulomatosis. J Clin Neuroophthalmol. 1985;5(1):17-25.

4. Gawley SD, McAvoy CE, Best RM, Flynn PA. Traumatic selfinduced orbital apex syndrome. Eye. 2007;21(11):1451-2.

5. Venkitaraman R, Madhavan J, Ramachandran K, Abraham E, Rajan B. Primary Adenoid Cystic Carcinoma Presenting as an Orbital Apex Tumor. Neuroophthalmology. 2008;32(1):27-32.

6. Marcet MM, Yang W, Albert DM, Salamat MS, Appen RE. Aspergillus infection of the orbital apex masquerading as TolosaHunt syndrome. Arch Ophthalmol. 2007;125(4):563-6.

7. Hughes EH, Petrushkin H, Sibtain NA, Stanford MR, Plant GT, Graham EM. Tuberculous orbital apex syndromes. Br J Ophthalmol. 2008;92(11):1511-7.

8. Kattah JC, Kennerdell JS. Orbital apex syndrome secondary to herpes zoster ophthalmicus. Am J Ophthalmol. 1978;85(3):378-82.

9. Marsh RJ, Cooper M. Ophthalmic herpes zoster. Eye. 1993;7 ( Pt 3):350-70.

10. Marsh RJ, Dulley B, Kelly V. External ocular motor palsies in ophthalmic zoster: a review. $\mathrm{Br} \mathrm{J}$ Ophthalmol. 1977;61(11):677-82.

11. Ramsell TG. Complications of herpes zoster ophthalmicus. Am J Ophthalmol. 1967 ;63(6):1796-8.

12. Wang AG, Liu JH, Hsu WM, Lee AF, Yen MY. Optic neuritis in herpes zoster ophthalmicus. Jpn J Ophthalmol. 2000;44(5):550-4.

13. Kawasaki A, Borruat FX. An unusual presentation of herpes zoster ophthalmicus: orbital myositis preceding vesicular eruption. Am J Ophthalmol. 2003;136(3):574-5.

14. Menon V, Kumar G, Tandon R. Optic neuropathy secondary to herpes zoster ophthalmicus. Indian J Ophthalmol. 1995;43(2):78-9.

15. Monteiro ML, Borges WI, do Val Ferreira Ramos C, Lucato LT, Leite CC. Bilateral optic neuritis in wegener granulomatosis. J Neuroophthalmol. 2005; 25(1):25-8.

\section{Endereço para correspondência: Mário Luiz Ribeiro Monteiro Av. Angélica 1757 cj 61 CEP 01227-200 - São Paulo (SP)}

E-mail: mlrmonteiro@terra.com.br 